symmetric space $V$ (irreducible or not) is again Hermitian symmetric and is isomorphic to $V$.

Proof. Since $H^{1}(V, \theta)=0$ [2], we see that the set of points $t \in B$ for which $V_{t}$ is isomorphic to $V$ is an open set in $B$ [3]; it is also closed by Theorem 2 .

\title{
REFERENCES
}

1. K. Srinivasacharyulu, Thesis to appear in Séminaire Ehresmann, Paris.

2. R. Bott, Homogeneous vector bundles, Ann. of Math. (2) 66 (1957), 203-248.

3. K. Kodaira and D. C. Spencer, On deformations of complex structures. I, II, Ann. of Math. (2) 67 (1958), 328-465.

UNIVERSITY OF MARYLAND AND

INSTITUTE FOR ADVANCED STUdy

\section{ON THE BEST APPROXIMATION FOR SINGULAR IN- TEGRALS BY LAPLACE-TRANSFORM METHODS}

\author{
BY HUBERT BERENS AND P. L. BUTZER \\ Communicated by Felix Browder, October 9, 1963
}

1. Introduction. Let $f(t)$ be a Lebesgue-integrable function in $(0, R)$ for every positive $R$. We denote by

$$
J_{\rho}(t)=\rho \int_{0}^{t} f(t-u) k(\rho u) d u
$$

a general singular integral with parameter $\rho>0$ and kernel $k$ having the following property $(\mathrm{P}): k(u) \geqq 0$ in $0 \leqq u<\infty, k \in L(0, \infty)$, and $\int_{0}^{\infty} k(u) d u=1$.

If we restrict the class of functions $f(t)$ such that $e^{-c t} f \in L_{p}(0, \infty)$, $1 \leqq p<\infty$, for every $c>0$, and if $k$ satisfies $(\mathrm{P})$, then the following statements hold:

(i) $J_{\rho}(t)$ exists as a function of $t$ almost everywhere, $e^{-c t} J_{\rho}$ $\in L_{p}(0, \infty)$ for every $c>0$, and $\left\|e^{-c t} J_{\rho}\right\|_{L_{p}(0, \infty)} \leqq\left\|e^{-c t} f\right\|_{L_{p}(0, \infty)}$;

(ii) $\lim _{\rho} \uparrow_{\infty}\left\|e^{-c t}\left\{f-J_{\rho}\right\}\right\|_{p}=0$.

Furthermore, we denote by

$$
\hat{f}(s)=\int_{0}^{\infty} e^{-s t} f(t) d t \quad(s=\sigma+i \tau, \operatorname{Re} s>0)
$$

the Laplace-transformation of a function $f$ belonging to one of the classes described above, and the Laplace-Stieltjes-transform of a 
function $h(t)$ locally of bounded variation at each $t \geqq 0$ with $\int_{0}^{\infty} e^{-c t}|d h(t)|<\infty$ for every $c>0$ by

$$
\check{h}(s)=\int_{0}^{\infty} e^{-s t} d h(t)
$$

$(\operatorname{Re} s>0)$.

Some fundamental hypotheses upon the kernel $k$ are needed to prove the approximation theorems stated below. Let $\hat{k}(s)(\operatorname{Re} s \geqq 0)$ be the Laplace-transform of $k$ : At first,

$$
\lim _{\rho \uparrow \infty}(s / \rho)^{-\gamma}[1-\hat{k}(s / \rho)]=A
$$

$(\operatorname{Re} s>0)$

should exist for some real $0<\gamma \leqq 1$, where $A$ is a positive finite constant; secondly, there exists a normalized function $Q(u)$ of bounded variation in $[0, \infty]$ with $Q(\infty)=1$ such that

$$
A^{-1}(s / \rho)^{-\gamma}[1-\hat{k}(s / \rho)]=\check{Q}(s / \rho) \quad(\operatorname{Re} s \geqq 0) ;
$$

and thirdly, let there be a $q \in L(0, \infty), \int_{0}^{\infty} q(u) d u=1$, and

$$
A^{-1}(s / \rho)^{-\gamma}[1-\hat{k}(s / \rho)]=\hat{q}(s / \rho) \quad(\operatorname{Re} s \geqq 0) .
$$

It may be mentioned here that the conditions (1.2) and (1.3), respectively, imply (1.1), but the inverse does not seem to hold. Moreover, if the kernel $k$ is not positive, then the constant $\gamma$ need not be bounded by one.

\section{Approximation theorems.}

THEOREM 1. Let $e^{-c t f}$, $e^{-c t} l \in L(0, \infty)$ for every $c>0$, let $k$ satisfy $(P)$, and let (1.1) hold for some real $\gamma(0<\gamma \leqq 1)$.

(i) Then $\left\|e^{-c t}\left\{\rho^{\gamma}\left(f-J_{\rho}\right)-l\right\}\right\|_{L_{1}(0, \infty)}=o(1)(\rho \uparrow \infty)$ implies

$$
A s^{\gamma} \hat{f}(s)=\imath(s)
$$

or

$$
f(t)=\frac{1}{A} \int_{0}^{t} \frac{(t-u)^{\gamma-1}}{\Gamma(\gamma)} l(u) d u \quad \text { a.e. }
$$

(ii) If $\left\|e^{-c t}\left\{f-J_{\rho}\right\}\right\|_{L_{1}(0, \infty)}=O\left(\rho^{-\gamma}\right)(\rho \uparrow \infty)$, then there exists a function $F(t)$ locally of bounded variation at $t \geqq 0$ with $\int_{0}^{\infty} e^{-c t}|d F(t)|<\infty$ for every $c>0$ such that

$$
A s^{\gamma} \hat{f}(s)=\check{F}(s)
$$

$(\operatorname{Re} s>0)$.

Sketch of Proof. As (i) can readily be shown, we will restrict ourselves to the proof of (ii), Clearly, 


$$
[1-k(s / \rho)] \hat{f}(s)=\int_{0}^{\infty} e^{-s t}\left\{f(t)-J_{\rho}(t)\right\} d t \quad(\operatorname{Re} s>0),
$$

and if we define

$$
\begin{array}{r}
S_{T}(t)=\frac{1}{2 \pi i} \int_{c-i T}^{c+i T}\left(1-\frac{|\tau|}{T}\right) e^{s t}[1-\hat{k}(s / \rho)] \hat{f}(s) d s \\
\quad(s=c+i \tau, c>0),
\end{array}
$$

then with the aid of the above equation it can be rewritten as

$$
S_{T}(t)=\frac{2}{\pi T} \int_{0}^{\infty} e^{c(t-u)} \frac{\sin ^{2}\{T(t-u) / 2\}}{(t-u)^{2}}\left\{f(u)-J_{\rho}(u)\right\} d u .
$$

Now, the large $O$-approximation of $f$ by $J_{\rho}$ gives

$$
\left\|e^{-c t} S_{T}\right\|_{L_{1}(-\infty, \infty)} \leqq\left\|e^{-o t}\left\{f-J_{\rho}\right\}\right\|_{L_{1}(0, \infty)}=O\left(\rho^{-\gamma}\right) \quad(\rho \uparrow \infty)
$$

for all $T \geqq 0$, and using the condition (1.1) and Lebesgue's dominated convergence theorem we have

$$
\frac{1}{2 \pi i} \int_{c \rightarrow i T}^{c+i T}\left(1-\frac{|\tau|}{T}\right) e^{s t} A s^{\gamma} \hat{f}(s) d s=\lim _{\rho \uparrow \infty} \rho^{\gamma} S_{T}(t),
$$

finally, with Fatou's lemma

$$
\begin{aligned}
\| e^{-c t} \frac{1}{2 \pi i} \int_{c-i T}^{c+i T}\left(1-\frac{|\tau|}{T}\right) e^{s t} A s^{\gamma} \hat{f}(s) d s & \|_{L_{1}(-\infty, \infty)} \\
& \leqq \liminf _{\rho \uparrow \infty} \rho^{\gamma}\left\|e^{-c t} S_{T}\right\|_{L_{1}(-\infty, \infty)}=O(1)
\end{aligned}
$$

for all $T \geqq 0$. Evidently the assumptions of the theorem and (1.1) give that $\left|A s^{\gamma} \hat{f}(s)\right|$ is uniformly bounded in $\operatorname{Re} s \geqq \delta>0$. Now, using a representation theorem for Laplace-transforms [1], this implies the existence of a function $F(t)$ such that (2.1) is valid.

The condition (2.1) defines a certain class $K$ of functions $f$, and Theorem 1 shows, if there exists a constant $\gamma$ such that (1.1) holds, and if $\left\|e^{-c t}\left\{f-J_{\rho}\right\}\right\|_{L_{1}(0, \infty)}=O\left(\rho^{-\gamma}\right)$, then $f \in K$. The next theorem now shows that the inverse holds too.

TheOREM 2. Let $e^{-c t} f \in L(0, \infty)$ for every $c>0$, let $k$ satisfy $(P)$, and let the relation (1.2) be satisfied for some $0<\gamma \leqq 1$. Then the assumption (2.1) implies $\left\|e^{-c t}\left\{f-J_{\rho}\right\}\right\|_{L_{1}(0, \infty)}=O\left(\rho^{-\gamma}\right)(\rho \uparrow \infty)$.

If the two foregoing theorems hold, we say the singular integral $J_{\rho}$ is said to be saturated with order $O\left(\rho^{-\gamma}\right)$, and the functions $f$ yielding 
(2.1) define the saturation class of $J_{\rho}$. It was J. Favard [5] who introduced this terminology in approximation theory, and one of the authors $[2 ; 3]$ first made use of Fourier-transform methods to determine the saturation classes of singular integrals which are convolution integrals connected with the Fourier-transform. This question was independently but a little later treated by G. Sunouchi [6] too. Now in this paper general singular integrals are discussed which are classical convolution integrals connected with the Laplace-transform. Although there are connections between the Fourier- and Laplacetransform methods, it may be mentioned that the special properties and peculiar structure of the Laplace-transform play an important role in the proofs and the formulations of the stated theorems.

In the space $L_{p}(0, \infty), 1<p<\infty$, an equivalent theorem holds too.

ThEOREM 3. Let $k$ satisfy $(P)$, and let the condition (1.3) exist for some constant $\gamma(0<\gamma \leqq 1)$. A necessary and sufficient condition that the singular integral $J_{\rho}(t)=\rho \int_{0}^{t} f(t-u) k(\rho u) d u(\rho>0)$ shall be saturated with order $O\left(\rho^{-\gamma}\right)$ for functions $e^{-c t} f \in L_{p}(0, \infty), 1<p<\infty, c>0$, is that there exists a function $e^{-c t} F \in L_{p}(0, \infty), c>0$, such that

$$
A s^{\gamma} \hat{f}(s)=\hat{F}(s)
$$

$(\operatorname{Re} s>0)$

or

$$
f(t)=\frac{1}{A} \int_{0}^{t} \frac{(t-u)^{\gamma-1}}{\Gamma(\gamma)} F(u) d u \quad \text { a.e. }
$$

3. Application. As an application we will consider a boundary value problem of heat conduction of a semi-infinite rod $(x \geqq 0)$. $U(x, t)$ is the temperature in the rod at time $t>0$, which is described by the equations

$$
\frac{\partial U(x, t)}{\partial t}=\frac{\partial^{2} U(x, t)}{\partial x^{2}}(x, t>0) ; \quad \lim _{x \downarrow 0} U(x, t)=U_{0}(t) \quad(t>0) .
$$

Among others, G. Doetsch [4, Bd. III] has shown that the solution is given by

$$
U(x, t)=\frac{x}{2 \sqrt{ } \pi} \int_{0}^{t} U_{0}(t-u) \frac{\exp \left(-x^{2} / 4 u\right)}{u^{3 / 2}} d u \quad(x, t>0),
$$

where $U_{0}$ is a Lebesgue-integrable function, and that the solution is unique, if the convergence of $U(x, t)$ to $U_{0}(t)$ is defined by the normconvergence of the given function space.

$U(x, t)$ is a singular convolution integral with parameter $\rho=1 / x^{2}>0$ and kernel 


$$
k(u)=\frac{1}{2 \sqrt{ } \pi} \frac{\exp (-1 / 4 u)}{u^{3 / 2}} \quad(0 \leqq u \leqq \infty) .
$$

It is easy to see that the kernel $k$ has property $(P)$ and its Laplacetransform $\hat{k}(s)=\exp (-\sqrt{ } s)$ satisfies the conditions (1.1), (1.2), and (1.3) for $\gamma=1 / 2$ with $A=1$.

If we restrict the temperature at the origin $U_{0}(t)$ such that $e^{-c t} U_{0}$ $\in L_{p}(0, \infty), 1<p<\infty, c>0$, for instance, then making use of Theorem 3 we have:

(i) $\left\|e^{-c t}\left\{U_{0}(t)-U(x, t)\right\}\right\|_{L_{p}(0, \infty)}=o(x)(c>0, x \downarrow 0)$ implies $U_{0}(t)=0$ a.e.;

(ii) $\left\|e^{-c t}\left\{U_{0}(t)-U(x, t)\right\}\right\|_{L_{p}(0, \infty)}=O(x)(c>0, x \downarrow 0)$ guarantees that the flux of heat at the boundary $W_{0}(t)$ exists a.e., $e^{-c t} W_{0} \in L_{p}(0, \infty)$ for every $c>0$, and

$$
\sqrt{ } s \hat{U}_{0}(s)=\hat{W}_{0}(s)
$$

or, equivalently,

$$
U_{0}(t)=\frac{1}{\sqrt{ } \pi} \int_{0}^{t} \frac{W_{0}(u)}{\sqrt{ } t-u} d u \text { a.e., }
$$

and vice versa.

The complete proofs of these and further results as well as a detailed discussion will appear elsewhere (see [7]).

\section{REFERENCES}

1. H. Berens und P. L. Butzer, Über die Darstellung holomorpher Funktionen durch Laplace- und Laplace-Stieltjes-Integrale, Math. Z. 81 (1963), 124-134.

2. P. L. Butzer, Sur le rôle de la transformation de Fourier dans quelques problèmes d'approximation, C. R. Acad. Sci. Paris 249 (1959), 2467-2469.

3. - Fourier-transform methods in the theory of approximation, Arch. Rational Mech. Anal. 5 (1960), 390-415.

4. G. Doetsch, Handbuch der Laplace-Transformation, Bd. III, Birkhäuser, Basel, 1950-1956.

5. J. Favard, Sur l'approximation des fonctions d'une variable réelle, Colloques Internationaux du Centre National de la Recherche Scientifique, No. 15, pp. 97-110. Centre National de la Recherche Scientifique, Paris, 1949.

6. G. Sunouchi, On the class of saturation in the theory of approximation. I, Tôhoku Math. J. 12 (1960), 339-344.

7. H. Berens and P. L. Butzer, On the best approximation for singular integrals by Laplace-transform methods, Proceedings of the Conference on Approximation, Oberwolfach, August, 1962, Birkhäuser, Basel (to appear).

The Technical University of Aachen 